\title{
Calidad de vida relacionada a la salud oral de preescolares peruanos en el año 2018
}

\author{
Jenny Elisa Maraví Gutiérrez, ${ }^{1}$ \\ Luis Angel Hayakawa Lastarria, ${ }^{2}$ \\ Dafna Geller Palti. ${ }^{3}$
}

\section{Resumen}

Antecedentes: Existen pocas investigaciones que muestren cómo la caries de aparición temprana (CAT) afecta a la mayoría de los niños peruanos. Sin embargo, es aún menor la cantidad de estudios que muestran cómo la CAT y la necesidad de tratamiento (NTx) afectan la calidad de vida de los menores. Objetivo: Evaluar el impacto de la salud bucal sobre la calidad de vida según CAT y NTx en preescolares del Perú. Materiales y métodos: Se realizó un estudio descriptivo-transversal conformado por una muestra de 360 niños de 3 a 5 años de una institución educativa pública del distrito de Independencia, en Lima. Los niños fueron examinados con el índice CEOD, NTx y el cuestionario de Escala de impacto de la salud oral en infancia temprana (ECOHIS). Los datos fueron analizados con estadística descriptiva y para relacionar se usó ANOVA y Kruskal Wallis $(p<0,05)$. Resultados: La prevalencia de CAT fue de $73,6 \%$ y la NTx más requerida fue la "obturación superficial". Se encontraron diferencias estadísticamente significativas tanto para CAT como para NTx en relación con todos los dominios de calidad de vida. Conclusiones: La CAT y la NTx tienen impacto negativo en la calidad de vida de los preescolares.

Palabras clave: Preescolar, calidad de vida, caries dental, prevalencia.

\footnotetext{
${ }_{1}^{1}$ Alumno de la Carrera de Odontología, Facultad de Ciencias de la Salud, Universidad Peruana de Ciencias Aplicadas-UPC, Lima, Perú. ${ }^{2}$ Cirujano dentista, egresado de la Universidad Peruana de Ciencias Aplicadas-UPC, Lima, Perú.

${ }^{3}$ Docente del área de Odontopediatría en la facultad de Odontología de la Universidad Peruana de Ciencias Aplicadas-UPC, Lima, Perú
} 
Artigo original

\section{Qualidade de vida relacionada à saúde bucal em pré-escolares peruanos em 2018}

\section{Resumo}

Contexto: Existem poucas pesquisas que demonstrem como a cárie precoce na infância (CPI) afeta a maioria das crianças peruanas. No entanto, é ainda menor o número de estudos que mostram como a CPI e a necessidade de tratamento (NTx) afetam a qualidade de vida das crianças. Objetivo: Avaliar o impacto da saúde bucal na qualidade de vida segundo a CPI e a NTx em pré-escolares do Peru. Material e métodos: Foi realizado um estudo descritivo-transversal, com uma amostra de 360 crianças de 3 a 5 anos de uma instituição de ensino público do distrito de Independência, em Lima. As crianças foram examinadas com o índice
CEOD, NTx e o questionário de a escala de impacto na saúde bucal na primeira infancia (ECOHIS). Os dados foram analisados com estatística descritiva e para relacioná-los foram usados ANOVA e Kruskal Wallis ( $p<0,05)$. Resultados: A prevalência de CPI foi de $73,6 \%$ e a NTx mais requerida foi a "obturação superficial". Foram encontradas diferenças estatisticamente significativas tanto para a CPI, quanto para a NTx em relação a todos os domínios da qualidade de vida. Conclusões: A CPI e a NTx tem impacto negativo na qualidade de vida dos pré-escolares.

Palavras chaves: Pré-escolar, qualidade de vida, cárie dentária, prevalência.

Original article

\section{Oral health-related quality of life in peruvian preschool children in 2018}

\begin{abstract}
Background: Few studies have investigated the effect of early childhood caries (ECC) on Peruvian children; there are even fewer studies of how ECC and treatment needs (TNs) affect the quality of life of these children. Objective: To assess the impact of oral health on the quality of life, according to ECC and TNs, of Peruvian preschool children. Materials and methods: A descriptive cross-sectional study was conducted, consisting of a sample of 360 children aged 3 to 5 years from a public educational institution in the district of Independencia, Lima. The children were examined using the decayed, missing,
\end{abstract}

and filled teeth (DMFT) index, the TNs index, and the early childhood oral health impact scale (ECOHIS) questionnaire. The data were analyzed with descriptive statistics using ANOVA and the KruskalWallis test to establish relationships $(\mathrm{p}<0.05)$. Results: The prevalence of ECC was $73.6 \%$, and the most required TN was "surface filling." Statistically significant differences were found for both ECC and TNs in relation to all quality of life domains. Conclusions: ECC and TNs have a negative impact on the quality of life of preschool children.

Key words: Dental caries, child, quality of life, prevalence. 


\section{Introducción}

El cuidado de la salud bucal, desde la niñez hasta la adultez, tiene un impacto significativo en la salud física y mental del individuo. ${ }^{1}$ Gozar de una adecuada salud bucodental en cualquier etapa de la vida es fundamental para un correcto desempeño de las actividades rutinarias. Es así que la calidad de vida relacionada con la salud (CVRS), o la salud percibida, integra aquellos aspectos de la vida directamente relacionados con el funcionamiento físico y mental y con el estado de bienestar de las personas. ${ }^{2-4}$

La calidad de vida y la condición oral de los individuos permanecieron por mucho tiempo desconocidas debido a que no existían instrumentos que pudieran ser aplicados en las diferentes edades. Sin embargo, en los últimos años se han desarrollado instrumentos validados en diferentes idiomas para poder evaluar la calidad de vida relacionada con la salud bucal. ${ }^{3,5}$

La salud bucal en el Perú, según un estudio epidemiológico realizado por el Ministerio de Salud (Minsa), muestra una prevalencia de caries dental de $85 \%$ en niños menores de 11 años, mientras que en aquellos de entre 3 y 5 años es de $76 \%{ }^{6}$ Debido a las altas cifras de prevalencia, en el año 2016 se aprobó el Plan Nacional de Salud Bucal, con diversas acciones para fomentar la prevención y promoción de la salud, y para garantizar el acceso a los servicios de salud oral; ${ }^{7}$ sin embargo, la población peruana aún sufre de este grave problema de salud que afecta a niños, adolescentes y adultos.

La caries dental es una alteración del equilibrio y la proporción entre las diferentes especies de microorganismos de la flora oral. Asimismo, es la disolución química localizada de la superficie dentaria como resultado de diferentes eventos metabólicos asociada a diversos factores, como la falta de higiene bucal, alimentación inadecuada, insuficiente información sobre medidas preventivas y falta de acceso a los servicios de salud. ${ }^{6,8-10}$

Una óptima salud oral es un componente esencial para la salud en general y la calidad de vida; es por ello que el Minsa y la AAPD recomiendan la atención odontológica a temprana edad.,6-8,10 Además, es de gran importancia identificar aquellos factores que afectan a la calidad de vida, tales como la severidad de la caries dental y la necesidad de tratamiento. ${ }^{10-13}$ Sin embargo, no existe suficiente evidencia científica confiable que demuestre su relación, sobre todo en la población peruana.

Por tal motivo, el propósito de este estudio fue evaluar la calidad de vida relacionada con la salud oral, la CAT y la necesidad de tratamiento en preescolares de 3 a 5 años de edad de la institución educativa pública William Fulbright del distrito de Independencia, en el año 2018.

\section{Materiales y métodos}

\section{Diseño del estudio}

El presente estudio fue de tipo analítico observacional, transversal. Se realizó en una institución educativa pública del distrito de Independencia, el cual es uno de los distritos en los que se concentran las poblaciones con mayor pobreza. Esta institución cuenta con un total de 450 
niños del nivel inicial entre las edades de 3 y 5 años.

\section{Población y muestra}

El tamaño de la muestra se determinó utilizando la fórmula de comparación de medias independientes, con un nivel de confianza de 95\%, precisión de 5\% y una potencia de $80 \%$; la información fue obtenida del estudio de López R. et al. en la provincia de Huaura, Lima. ${ }^{13}$ Esto se realizó con el objetivo de establecer un número mínimo de preescolares que debían ser evaluados para que el presente estudio tuviera relevancia significativa. Posteriormente se realizó un censo con el fin de abarcar a toda la población; sin embargo, debidoalos criterios deexclusión, solo se evaluaron 360 estudiantes, ya que 58 no tenían consentimiento informado, 20 no completaron los cuestionarios, 7 no colaboraron y 5 se retiraron.

\section{Criterios de selección}

\section{Criterios de inclusión}

1. Niños matriculados en el instituto educativo William Fulbright del distrito de Independencia.

2. Niños que se encuentren dentro del rango de 3 a 5 años de edad.

3. Niños de ambos sexos.

4. Niños cuyos padres o apoderados hayan firmado un consentimiento informado.

\section{Criterios de exclusión}

1. Niños con discapacidad, pues la ejecutora del presente estudio no ha recibido el entrenamiento especializado adecuado para evaluar a niños con discapacidad.

2. Niños cuyos padres no hayan firmado el consentimiento informado.

3. Niños que no cooperen con la evaluación bucodental, por ejemplo, niños con ansiedad, niños que se rehúsen a participar, que manifiesten incomodidad mediante el llanto o gritos que puedan perturbar a los demás menores, etcétera.

\section{Técnicas y procedimientos}

\section{Coordinaciones previas}

Se realizaron los trámites y las coordinaciones correspondientes con la institución educativa. Posteriormente se realizó una reunión con los padres de familia, en la cual se les explicó detalladamente el proceso del estudio y se procedió a distribuir un sobre con el consentimiento informado, el cuestionario ECOHIS, la encuesta del nivel socioeconómico y un bolígrafo (Figuras 1 y 2). Se sugirió que firmaran el consentimiento informado y que luego completaran el cuestionario y las encuestas, mediante lo cual, estaban autorizando la participación de su hijo menor. Una vez llenados los documentos, se les proporcionó dípticos informativos sobre la CAT temprana y salud bucal.

\section{Capacitación y calibración}

Para la evaluación de la CAT y la necesidad de tratamiento se realizó una calibración sobre el índice CEOD y NTx inter e intrasujetos con el especialista en odontopediatría con 20 imágenes de fotografías clínicas y se obtuvo un índice 
CALIDAD DE VIDA

CUESTIONARIO EARLY CHILDHOOD ORAL HEALTH MMPACT SCALE (ECOHIS)

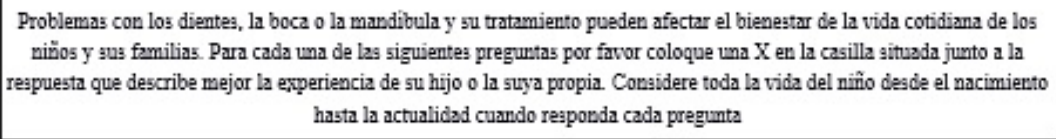

1.1 IMPACTO DE LOS PROBLEMAS BUCALES CON EL NINO
1.1.1 Con qué frecuencia su hijo ha tenido Dolor en los dientes, boca o mandibula?
Nunca Casi nunca Ocasionalmente A menudo Muy a menudo No sabe/ no contesta
$\begin{array}{llllll}0 & O_{1} & \mathrm{O}_{2} & \mathrm{O}_{3} & \mathrm{O}^{4} & \end{array}$
1.1.2 Con qué frecuencia su hijo ha tenido dificultades para tomar bebidas calientes o frias, debido a problemas dentales o tratanientos odontológicos?

$\begin{array}{cccccc}\text { Nunca } & \text { Casi nunca } & \text { Ocasionalmente } & \text { A menudo } & \text { Muy a menudo } & \text { No sabe/ no conteata } \\ \text { O0 } & \mathrm{O}_{1} & \mathrm{O}_{2} & \mathrm{O}_{3} & \mathrm{O}^{4} & \mathrm{O}_{5}\end{array}$

1.1.3 Con qué frecuencia su hijo ha tenido dificultades para comer algunos alimentos a causa de problemas dentales o tratamientos odoutológicos?

$\begin{array}{llllll}\text { Nunca } & \text { Casi nunca } & \text { Ocasionalmente } & \text { A menudo } & \text { Muy a menudo } & \text { No sabe/ no conteata } \\ \text { O } & \mathrm{O}_{1} & \mathrm{O}_{2} & \mathrm{O}_{3} & \mathrm{O}_{4} & \mathrm{O}_{5}\end{array}$

$1 \mathrm{~T} 4$ iCon qué frecuencia su hijo ha tenido dificultades para pronumciar algunas palabras, por problemas dentales o tratamientos odoutologicos?
Nunca Casi nunca
O० 01
102
A menudo
Muy a menu
No sabe' no contesta

1.1 .5 ¿Con que frecuencia su hijo ha perdido dias de asistencia a su actividad preescolar, de guardería o escuela por problemas dentales o tratamientos odontológicos?

$\begin{array}{cccccc}\text { Nurca } & \text { Casi nunca } & \text { Ocasionalmente } & \text { A menudo } & \text { Muy a menudo } & \text { No sabe no conteata } \\ \text { O0 } & \mathrm{O}_{1} & \mathrm{O}_{2} & \mathrm{O}_{3} & \mathrm{O}_{4} & \text { S }_{5}\end{array}$

1.1.6 ¿Con qué frecuencia su hijo ba tenido problemas para dormir debido a problemas dentales o tratamientos odontologicos?

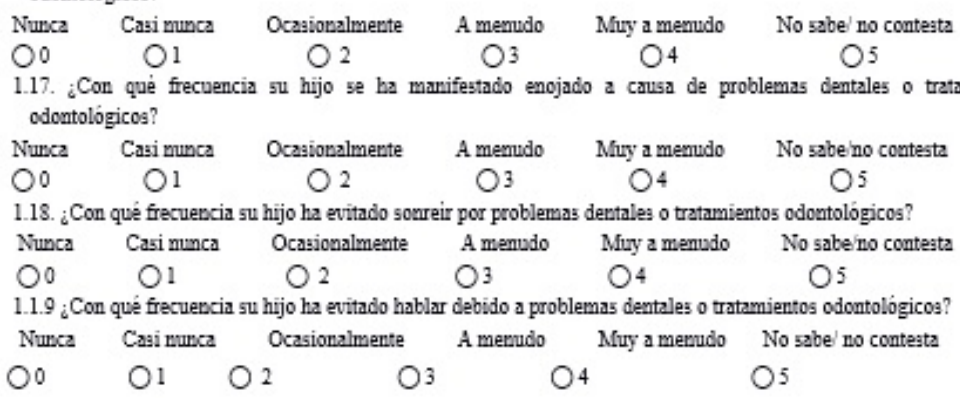

\subsection{INPACTO DELOS PROBLEMAS BUCALES CON BL PADRE O FAMILIAR}

1.2.1 Con que frecuencia usted u otro miembro de la familia se ha preocupedo a causa de problemas dentales o tratamientos odontológicos de su hijo?

$\begin{array}{cccccc}\text { Nunca } & \text { Casi nunca } & \text { Ocasionalmente } & \text { A menudo } & \text { Muy a menudo } & \text { No sabe/ no contesta } \\ \text { O0 } & \mathrm{O}_{1} & \mathrm{O}_{2} & \mathrm{O}_{3} & \mathrm{O}_{4} & \mathrm{~S}_{5}\end{array}$

1.2 .2 ¿Con qué frecuencia usted u otro miembro de la familia se ha sentido culpable por los problemas dentales o tratamientos odontológicos de su hijo?
Nunce
Ocasionalmente
A menudo
Muy a menudo
No sabe/ no contesta
Oo 01
○ 2
O3
O4
O5

1.2 .3 ¿Con qué frecuencia usted u otro miembro de la familia ha ocupado el tiempo de trabajo o actividad a causa de los problemsas dertales o tratamientos odontológicos de su hijo?

\begin{tabular}{|c|c|c|c|c|c|}
\hline Nunca & Casi nunca & Ocasionalmente & A menudo & Muy a menudo & No sabe no contesta \\
\hline 00 & 01 & 02 & $\mathrm{O}^{3}$ & $\mathrm{O}_{4}$ & $\mathrm{O} 5$ \\
\hline \multicolumn{6}{|c|}{$\begin{array}{l}1.2 .4 \text { ¿Con que frecuencia los problemas dentales o tratamientos odontológicos de su hijo han afectado a la economia } \\
\text { de su hogar? }\end{array}$} \\
\hline Nunca & Casi nunca & Ocasionalmente & A menudo & Muy a menudo & No sabe/ no conteata \\
\hline 00 & 01 & 02 & $\mathrm{O}_{3}$ & $\mathrm{O}_{4}$ & $\mathrm{O} 5$ \\
\hline
\end{tabular}

Figura 1: Early childhood oral health impact scale (ECHOHIS) 
1. ¿Cuál es el grado de estudios de los padres? Marque con un aspa (X) para ambos padres; M= Madre y $\mathrm{P}=$ Padre.

1 Primaria completa / incompleta

2 Secundaria incompleta

3 Secundaria completa

4 Superior no universitario (por ejemplo, instituto superior)

5 Estudios universitarios incompletos

6 Estudios universitarios completos (bachiller, titulado)

7 Posgrado

2. ¿A dónde acude el jefe de hogar para atención médica cuando tiene algún problema de salud?

1 Posta médica / farmacia / naturista

2 Hospital del Ministerio de Salud / Hospital de la Solidaridad

3 Seguro social / Hospital FFAA / Hospital de Policía

4 Médico particular en consultorio

5 Médico particular en clínica privada

3. ¿Cuál de las siguientes categorías se aproxima más al salario mensual de su casa?

1 Menos de 750 soles/mes aproximadamente

2 Entre 750 y 1000 soles/mes aproximadamente

3 Entre 1001 y 1500 soles/mes aproximadamente

4 Más de 1500 soles/mes aproximadamente

4. ¿Cuántas habitaciones tiene su hogar exclusivamente para dormir?

1. 1

2. 2

3. 3

4. 4

5. más de 5

5 ¿Cuántas personas viven permanentemente en el hogar (sin incluir el servicio doméstico)?

1. 2

2. 3

3. 4

4. 5

5. 6

6. 7

7. más de 8

6.- ¿Cuál es el material predominante en los pisos de su vivienda?

1 Tierra / arena

2 Cemento sin pulir (falso piso)

3 Cemento pulido / tapizón

4 Mayólica / loseta / cerámicos

5 Parqué / madera pulida / alfombra / mármol / terrazo

Figura 2: Cuestionario de nivel socioeconómico versión modificada 2011-2012 (Perú). 
Kappa de Cohen de 0,86, lo que resulta una fuerza de concordancia "muy buena".

\section{Evaluación de las variables de estudio}

La evaluación clínica y el diagnóstico de CAT se realizó en un lugar previsto por la institución educativa, donde se colocaron todos los materiales e instrumentos necesarios en una mesa para la exploración. Los estudiantes fueron trasladados del salón de clases al área de revisión por el ayudante del profesor del salón correspondiente. Se llevó a los menores a lavarse los dientes con la asistencia del investigador para colaborar con la instrucción del correcto proceso para una buena higiene oral. Cabe mencionar que a los padres de familia y apoderados que no acudieron a la reunión convocada se les atendió los días de la ejecución del proyecto, y se les explicó el estudio. Asimismo, se les proporcionó un sobre con los documentos pertinentes respecto del estudio, donde se les pidió completar y firmar el consentimiento informado, la encuesta del nivel socioeconómico ${ }^{14}$ y el cuestionario ECOHIS. ${ }^{15}$

El cuestionario ECOHIS comprende 13 preguntas divididas en dos secciones: el impacto de este fenómeno en el niño y el impacto acontecido en la familia. La primera consta de nueve preguntas que se dividen en cuatro dimensiones o dominios, y la segunda tiene un total de cuatro preguntas distribuidas en dos dominios, y otorgan un puntaje final según lo respondido. Las 13 preguntas tuvieron un puntaje de 0 a 5 , siendo nunca, casi nunca, ocasionalmente, a menudo y muy a menudo; por su parte, es no sabe/ no contesta (Figura 1). Posteriormente, las puntuaciones de cada pregunta fueron sumadas y divididas para encontrar el puntaje promedio, y seguido de esto se procedió a sumar todas las puntuaciones promediadas de cada pregunta para hallar una puntuación promedio final, la cual podía resultar en un impacto positivo o negativo en la calidad de vida del menor. ${ }^{15-18}$

Luego de que los padres completaron el cuestionario y la encuesta, se les proporcionó dípticos informativos sobre la CAT y salud bucal y se procedió a continuar con el proceso de examinación. Se sentó a los menores en una silla uno por uno y se les observaron las piezas dentales secándolas con un algodón y haciendo uso del equipo de examen. Se utilizó además un frontoluz para asegurar una correcta iluminación. Asimismo, se utilizaron todas las medidas de bioseguridad y protección personal tanto para el operador como para el estudiante.

En el examen bucal se evaluó la experiencia de caries dental utilizando el índice CEOD (cariados, extraídos y obturados), así como el criterio de la American Academy of Pediatrics Dentristy (AAPD) para denominar la enfermedad de CAT (Tabla 1). Se procedió a evaluar CAT secando con una torunda de algodón las superficies dentarias para de esta manera determinar la presencia de una o más lesiones cariosas, pérdidas de piezas dentarias por caries $\mathrm{y}$ restauraciones de cualquier diente primario. Por otro lado, se consideró CAT severa cualquier señal de caries en una superficie lisa de dientes antero-superiores, pérdidas de piezas dentarias por caries o restauraciones en niños menores de 3 años que sumadas excedan la edad del paciente: 4 caries para niños de 3 años, 5 para los de 4 años y 6 para los de 5 años. ${ }^{8}$ 
Tabla 1. Códigos de CEOD.

\begin{tabular}{|c|c|}
\hline \multicolumn{2}{|c|}{ Códigos CEOD } \\
\hline Código & Condición \\
\hline A & Sano \\
\hline B & Cariado \\
\hline C & Obturado con caries \\
\hline D & Obturado sin caries \\
\hline E & Perdido por caries \\
\hline F & Sellante o fisura obturada \\
\hline
\end{tabular}

Posteriormente, se registró la necesidad de tratamiento utilizando los códigos de la Organización Mundial de la Salud, con la posibilidad de colocar desde un número 0 hasta un 9 con respecto al índice ${ }^{19}$ (Tabla 2). Estos valores fueron registrados en una ficha epidemiológica para la futura base de datos del proyecto. Terminado el examen oral los menores fueron regresados a sus salones acompañados por el asistente del profesor.

Tabla 2. Códigos de la necesidad de tratamiento según OMS (1997)

\begin{tabular}{|c|c|}
\hline Código & Necesidad de tratamiento (NTx) \\
\hline 0 & Ninguno \\
\hline $\mathrm{P}$ & Preventivo \\
\hline F & Obturación de fisura \\
\hline 1 & Obturación superficial \\
\hline 2 & $\begin{array}{c}\text { Dos o más obturaciones } \\
\text { superficiales }\end{array}$ \\
\hline 3 & Corona por cualquier motivo \\
\hline 4 & Revestimiento o lamina \\
\hline 5 & $\begin{array}{l}\text { Cuidado de la pulpa y } \\
\text { restauración }\end{array}$ \\
\hline 6 & Extracción \\
\hline 9 & No registrado \\
\hline
\end{tabular}

Plan de análisis

La base de datos se realizó en el programa Microsoft Excel y se analizaron los resultados mediante el paquete estadístico SPSS ${ }^{\circledR}$ versión 21.0 .

Para el análisis univariado se procedió a obtener la estadística descriptiva, la frecuencia absoluta y relativa de la variable CAT, la necesidad de tratamiento, la calidad de vida según ECOHIS, el nivel socioeconómico, el sexo y la edad.

Para el análisis bivariado se aplicó la prueba de ANOVA para relacionar el total de la calidad de vida con la CAT y la necesidad de tratamiento. Además, se usó la prueba de Kruskal Wallis para relacionar calidad de vida con CAT, necesidad de tratamiento, edad, sexo y nivel socioeconómico.

\section{Consideraciones éticas}

En primera instancia se solicitó la autorización del Comité de Ética de la Universidad Peruana de Ciencias Aplicadas para la ejecución del presente proyecto, obteniendo como respuesta una carta de aprobación con el número FCS/336-03-18. Asimismo, para el consentimiento de los participantes y sus apoderados se les solicitó una firma en señal de su aceptación y participación en el estudio, de forma previa a la recolección de datos.

\section{Resultados}

En el presente estudio la muestra estuvo constituida por 360 niños entre las edades de 3,4 y 5 años del sexo femenino y 
masculino. Esta población pertenecía en su mayoría al nivel socioeconómico $\mathrm{C}$, con un porcentaje de 53,1\% (Tabla 3).

Tabla 3. Prevalencia de caries de aparición temprana y distribución de la necesidad de tratamiento, nivel socioeconómico, sexo y edad en niños de 3 a 5 años de edad en una institución educativa pública del Perú.

\begin{tabular}{|c|c|c|}
\hline & $\mathbf{N}$ & $\%$ \\
\hline \multicolumn{3}{|l|}{ EDAD } \\
\hline 3 años & 113 & 31,4 \\
\hline 4 años & 116 & 32,2 \\
\hline 5 años & 131 & 36,4 \\
\hline \multicolumn{3}{|l|}{ GÉNERO } \\
\hline Masculino & 185 & 51,4 \\
\hline Femenino & 175 & 48,6 \\
\hline \multicolumn{3}{|l|}{ NIVEL SOCIOECONÓMICO } \\
\hline Nivel A & - & - \\
\hline Nivel B & 14 & 3,9 \\
\hline Nivel C & 191 & 53,1 \\
\hline Nivel D & 155 & 43,1 \\
\hline Nivel E & - & - \\
\hline \multicolumn{3}{|c|}{ CARIES DE APARICIÓN TEMPRANA } \\
\hline Sano & 95 & 26,4 \\
\hline $\begin{array}{l}\text { Caries de aparición } \\
\text { temprana }\end{array}$ & 130 & 36,1 \\
\hline $\begin{array}{l}\text { Caries de aparición } \\
\text { temprana severa }\end{array}$ & 135 & 37,5 \\
\hline \multicolumn{3}{|c|}{ NECESIDAD DE TRATAMIENTO } \\
\hline Una obturación superficial & 212 & 58,9 \\
\hline $\begin{array}{l}\text { Dos o más obturaciones } \\
\text { superficiales }\end{array}$ & 34 & 9,4 \\
\hline $\begin{array}{l}\text { Corona por cualquier } \\
\text { motivo }\end{array}$ & 4 & 1,1 \\
\hline Revestimiento o lámina & - & - \\
\hline $\begin{array}{l}\text { Cuidado de la pulpa y } \\
\text { restauración }\end{array}$ & 8 & 2,2 \\
\hline Extracción & 7 & 1,9 \\
\hline Preventivo/Sellante & 95 & 26,4 \\
\hline
\end{tabular}

La prevalencia de CAT fue de $73,6 \%$ de la población evaluada. Asimismo, se determinó que la necesidad de tratamiento más requerida por la población fue "una obturación superficial”, con 58,9\% (Tabla 3).

En cuanto a la encuesta de ECOHIS para medir el impacto en la calidad de vida, se encontró que la preocupación de los padres fue el problema más destacado como impacto en la familia, con un 56,9\%. Por otro lado, con respecto al impacto en el niño, la opción "ocasionalmente/a menudo/muy a menudo" tuvo una mayor distribución en la pregunta sobre dolor en los dientes, boca o mandíbula, con 18,3\%. Por último, el evitar hablar por parte del niño ha sido lo menos común, siendo marcado por los padres como "nunca/ casi nunca", situación similar al factor de gasto económico para la familia. A su vez, no se encontraron valores en la opción "no sabe" tanto en el impacto en el niño como en la familia (Tabla 4).

Al evaluar la CAT sobre la calidad de vida se encontró que el dominio "Limitaciones funcionales" fue en el que la enfermedad tuvo un mayor impacto sobre los niños, con un promedio de $1,22 \pm 1,82$ para CAT y de $2,27 \pm 2,55$ para CATs. Por otro lado, con respecto al impacto en la familia, se obtuvo que la "angustia de los padres" fue el dominio más afectado, con una media de 3,35 $\pm 2,44$ y de 2,72 $\pm 2,28$ para CAT, encontrándose significancia estadística en ambos casos $(p<0,05)$. Esto se puede ver en la Tabla 5 .

En lo que refiere a la necesidad de tratamiento sobre la calidad de vida, el mayor impacto en el niño se observa en el dominio "Limitaciones funcionales", en el que el tratamiento con mayor promedio es 
Tabla 4. Escala de impacto sobre la calidad de vida relacionada a la salud bucal de niños de 3 a 5 años de edad de una institución educativa pública del Perú.

\begin{tabular}{|l|c|c|c|}
\hline \multirow{2}{*}{$\begin{array}{l}\text { ESCALA DE IMPACTO EN LA SALUD BUCAL EN } \\
\text { LA INFANCIA TEMPRANA - ECOHIS }\end{array}$} & $\begin{array}{c}\text { Nunca / } \\
\text { Casi nunca }\end{array}$ & $\begin{array}{c}\text { Ocasionalmente/ } \\
\text { A menudo/ } \\
\text { Muy menudo }\end{array}$ & No sabe \\
\cline { 2 - 4 } & $\mathbf{n}(\%)$ & $\mathbf{n}(\%)$ & $\mathbf{n} \%$ \\
\hline IMPACTO EN EL NIÑO & $294(81,7)$ & $66(18,3)$ & $0(0,0)$ \\
\hline 1. Dolor en los dientes, boca o mandíbula & $318(88,3)$ & $42(11,7)$ & $0(0,0)$ \\
\hline 2. Dificultades para tomar bebidas calientes o frías & $301(83,6)$ & $59(16,4)$ & $0(0,0)$ \\
\hline 3. Dificultades para comer algunos alimentos & $317(88,1)$ & $43(11,9)$ & $0(0,0)$ \\
\hline 4. Dificultades para pronunciar algunas palabras & $340(94,4)$ & $20(5,6)$ & $0(0,0)$ \\
\hline $\begin{array}{l}\text { 5. Perdida de días de asistencia a su actividad } \\
\text { preescolar, guardería o escuela. }\end{array}$ & $334(92,8)$ & $26(7,2)$ & $0(0,0)$ \\
\hline $\begin{array}{l}\text { 6. Problemas para dormir } \\
\text { 7. Ha estado enojado o frustrado }\end{array}$ & $316(87,8)$ & $44(12,2)$ & $0(0,0)$ \\
\hline 8. Ha evitado sonreír & $344(95,6)$ & $16(4,4)$ & $0(0,0)$ \\
\hline 9. Ha evitado hablar & $342(95,0)$ & $18(5,0)$ & $0(0,0)$ \\
\hline IMPACTO EN LA FAMILIA & $155(43,1)$ & $205(56,9)$ & $0(0,0)$ \\
\hline $\begin{array}{l}\text { 10. Usted u otro miembro de la familia ha estado } \\
\text { preocupado }\end{array}$ & $234(65)$ & $126(35,0)$ & $0(0,0)$ \\
\hline $\begin{array}{l}\text { 11. Usted u otro miembro de la familia se ha sentido } \\
\text { culpable }\end{array}$ & $290(80,6)$ & $70(19,4)$ & $0(0,0)$ \\
\hline $\begin{array}{l}\text { 12. Usted u otro miembro de la familia ha ocupado } \\
\text { tiempo }\end{array}$ & $98(27,2)$ & $0(0,0)$ \\
\hline \begin{tabular}{l} 
13.Han tenido un impacto económico en su familia \\
\hline
\end{tabular} & & & \\
\hline
\end{tabular}

la extracción, con una media de $3,57 \pm 2,23$; con respecto al impacto en la familia, se observa también que las extracciones son las que afectan más, ubicándose en el dominio "Angustia de los padres" con un $4,86 \pm 2,34$. Para ambos datos se encontró significancia estadística de $\mathrm{p}<0,05$ (Tabla 5).

Por otro lado, al relacionar edad, sexo y nivel socioeconómico con calidad de vida se encontró que los niños con el promedio de puntaje ECOHIS más alto fueron los de

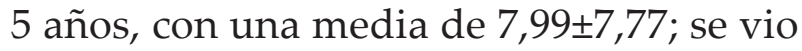
preponderancia en el sexo femenino, con $7,55 \pm 7,52$, y en el nivel socioeconómico B, con $8,21 \pm 6,54$. Sin embargo, no se halló una relación directa entre estos factores ya que no hubo significancia estadística (Tabla 6). 
Tabla 5. Impacto de la salud bucal sobre la calidad de vida según caries de aparición temprana y necesidad de tratamiento en niños de 3 a 5 años de una institución educativa pública del Perú.

\begin{tabular}{|c|c|c|c|c|c|c|c|c|}
\hline & \multirow[b]{3}{*}{$n(\%)$} & \multicolumn{7}{|c|}{ CALIDAD DE VIDA EN LA SALUD BUCAL SEGÚN DOMINIOS } \\
\hline & & \multicolumn{4}{|c|}{ IMPACTO EN EL NIÑO } & \multicolumn{3}{|c|}{ IMPACTO EN LA FAMILIA } \\
\hline & & $\begin{array}{l}\text { Síntomas } \\
\text { orales } \\
\text { X(D.S) }\end{array}$ & $\begin{array}{c}\text { Limitación } \\
\text { funcionales } \\
\text { X(D.S) }\end{array}$ & $\begin{array}{c}\text { Aspectos } \\
\text { psicológicos } \\
\text { X(D.S) }\end{array}$ & $\begin{array}{c}\text { Auto } \\
\text { imagen/ } \\
\text { interacción } \\
\text { social X(D.S) }\end{array}$ & $\begin{array}{l}\text { Angustia } \\
\text { de los } \\
\text { padres } \\
\text { X(D.S) }\end{array}$ & $\begin{array}{l}\text { Función } \\
\text { familia } \\
\text { X(D.S) }\end{array}$ & $\begin{array}{l}\text { Total } \\
\text { ECOHIS } \\
\text { X(D.S) }\end{array}$ \\
\hline \multicolumn{9}{|c|}{ CARIES DE APARICIÓN TEMPRANA } \\
\hline Sano & $95(26,4)$ & $0,41(0,68)$ & $0,96(1,84)$ & $0,40(0,90)$ & $0,21(0,87)$ & $2,04(1,95)$ & $1,24(1,63)$ & $5,26(5,59)$ \\
\hline $\begin{array}{l}\text { Caries de } \\
\text { aparición } \\
\text { temprana }\end{array}$ & $130(36,1)$ & $0,48(0,73)$ & $1,22(1,82)$ & $0,38(0,94)$ & $0,22(0,58)$ & $2,72(2,28)$ & $1,26(1,53)$ & $6,28(5,77)$ \\
\hline $\begin{array}{l}\text { Caries de } \\
\text { aparición } \\
\text { temprana } \\
\text { severa }\end{array}$ & $135(37,5)$ & $0,84(0,98)$ & $2,27(2,55)$ & $0,97(1,47)$ & $0,53(1,16)$ & $3,35(2,44)$ & $1,81(1,81)$ & $9,79(8,48)$ \\
\hline Valor P & & $<0,001$ & $<0,001$ & $<0,001$ & 0,002 & $<0,001$ & 0,009 & $<0,001^{*}$ \\
\hline \multicolumn{9}{|c|}{ NECESIDAD DE TRATAMIENTO } \\
\hline $\begin{array}{l}\text { Una ob- } \\
\text { turación } \\
\text { Superficial }\end{array}$ & $212(58,9)$ & $0,60(0,85)$ & $1,61(2,23)$ & $0,59(1,19)$ & $0,35(0,97)$ & $2,91(2,34)$ & $1,46(1,70)$ & $7,53(7,27)$ \\
\hline $\begin{array}{l}\text { Dos o más } \\
\text { obturaciones } \\
\text { superficiales }\end{array}$ & $34(9,4)$ & $0,76(0,85)$ & $1,97(1,82)$ & $0,71(1,22)$ & $0,26(0,51)$ & $3,29(2,37)$ & $1,68(1,47)$ & $8,68(6,05)$ \\
\hline $\begin{array}{l}\text { Corona por } \\
\text { cualquier } \\
\text { motivo }\end{array}$ & $4(1,1)$ & $1,50(1,29)$ & $2,75(4,86)$ & $1,75(2,36)$ & $0,50(1,00)$ & $2,50(3,00)$ & $1,50(2,38)$ & $10,50(14,39)$ \\
\hline $\begin{array}{l}\text { Revesti- } \\
\text { miento o } \\
\text { lámina } \\
\end{array}$ & - & - & - & - & - & - & - & - \\
\hline $\begin{array}{l}\text { Cuidados de } \\
\text { la pulpa y } \\
\text { restauración }\end{array}$ & $8(2,2)$ & $1,00(0,93)$ & $2,63(3,34)$ & $1,00(1,51)$ & $0,63(0,92)$ & $4,00(2,98)$ & $1,88(2,23)$ & $11,13(10,01)$ \\
\hline Extracción & $7(1,9)$ & $1,29(1,25)$ & $3,57(2,23)$ & $2,43(1,72)$ & $1,29(1,11)$ & $4,86(2,34)$ & $3,00(1,41)$ & $16,43(8,10)$ \\
\hline $\begin{array}{l}\text { Prevención/ } \\
\text { Sellante }\end{array}$ & $95(26,4)$ & $0,41(0,68)$ & $0,96(1,84)$ & $0,40(0,90)$ & $0,21(0,87)$ & $2,04(1,95)$ & $1,24(1,63)$ & $5,26(5,59)$ \\
\hline Valor P & & 0,017 & 0,002 & 0,002 & $<0,001$ & 0,002 & 0,073 & $<0,001^{*}$ \\
\hline
\end{tabular}

Prueba de Kruskal-Wallis $(p<0,05)$

*Prueba Anova $(p<0,05)$

$X=$ Media 
Tabla 6. Impacto de la salud bucal sobre la calidad de vida según la edad, género y nivel socioeconómico en niños de 3 a 5 años en una institución educativa pública del Perú

\begin{tabular}{|c|c|c|c|c|c|c|c|c|}
\hline \multirow{3}{*}{ 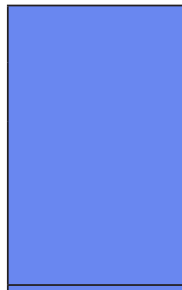 } & \multirow{3}{*}{$\mathrm{n}(\%)$} & \multicolumn{7}{|c|}{ CALIDAD DE VIDA EN LA SALUD BUCAL SEGÚN DOMINIOS } \\
\hline & & \multicolumn{4}{|c|}{ IMPACTO EN EL NIÑO } & \multicolumn{3}{|c|}{ IMPACTO EN LA FAMILIA } \\
\hline & & $\begin{array}{c}\text { Síntomas } \\
\text { orales } \\
\text { X(D.S) }\end{array}$ & $\begin{array}{l}\text { Limitación } \\
\text { funcionales } \\
\text { X(D.S) }\end{array}$ & $\begin{array}{c}\text { Aspectos } \\
\text { psicológicos } \\
\text { X(D.S) }\end{array}$ & $\begin{array}{c}\text { Auto } \\
\text { imagen/ } \\
\text { interacción } \\
\text { social X(D.S) } \\
\end{array}$ & $\begin{array}{c}\text { Angustia } \\
\text { de los } \\
\text { padres } \\
\text { X(D.S) } \\
\end{array}$ & $\begin{array}{l}\text { Función } \\
\text { familia } \\
\text { X(D.S) }\end{array}$ & $\begin{array}{l}\text { Total } \\
\text { ECOHIS } \\
\text { X(D.S) }\end{array}$ \\
\hline \multicolumn{9}{|l|}{ EDAD } \\
\hline 3 años & $113(31,4)$ & $0,56(0,80)$ & $1,37(1,90)$ & $0,50(0,99)$ & $0,18(0,50)$ & $2,82(2,11)$ & $1,47(1,76)$ & $6,89(5,89)$ \\
\hline 4 años & $116(32,2)$ & $0,56(0,82)$ & $1,44(2,24)$ & $0,50(1,05)$ & $0,41(1,10)$ & $2,59(2,40)$ & $1,48(1,70)$ & $6,99(7,47)$ \\
\hline 5 años & $131(36,4)$ & $0,67(0,89)$ & $1,79(2,39)$ & $0,80(1,42)$ & $0,40(1,01)$ & $2,89(2,41)$ & $1,44(1,62)$ & $7,99(7,77)$ \\
\hline Valor P & & 0,616 & 0,486 & 0,297 & 0,257 & 0,517 & 0,981 & 0,601 \\
\hline \multicolumn{9}{|l|}{ GÉNERO } \\
\hline Masculino & $185(51,4)$ & $0,54(0,81)$ & $1,43(2,00)$ & $0,55(1,04)$ & $0,35(0,96)$ & $2,76(2,29)$ & $1,47(1,62)$ & $7,11(6,75)$ \\
\hline Femenino & $175(48,6)$ & $0,66(0,87)$ & $1,66(2,39)$ & $0,67(1,33)$ & $0,31(0,88)$ & $2,79(2,34)$ & $1,46(1,75)$ & $7,55(7,52)$ \\
\hline Valor P & & 0,171 & 0,772 & 0,959 & 0,723 & 0,959 & 0,731 & 0,847 \\
\hline \multicolumn{9}{|c|}{ NIVEL SOCIO ECONOMICO } \\
\hline Nivel A & - & - & - & - & - & - & - & - \\
\hline Nivel B & $14(3,9)$ & $0,57(0,85)$ & $1,93(2,09)$ & $0,71(1,33)$ & $0,07(0,27)$ & $3,43(2,21)$ & $1,50(1,16)$ & $8,21(6,54)$ \\
\hline Nivel C & $191(53,1)$ & $0,59(0,84)$ & $1,32(2,04)$ & $0,52(1,09)$ & $0,24(0,69)$ & $2,64(2,19)$ & $1,35(1,60)$ & $6,66(6,54)$ \\
\hline Nivel D & $155(43,1)$ & $0,62(0,84)$ & $1,79(2,37)$ & $0,71(1,28)$ & $0,47(1,16)$ & $2,88(2,47)$ & $1,60(1,82)$ & $8,06(7,80)$ \\
\hline Nivel E & - & - & - & - & - & - & - & - \\
\hline Valor P & & 0,928 & 0,131 & 0,292 & 0,032 & 0,412 & 0,443 & 0,261 \\
\hline
\end{tabular}

\section{Discusión}

En el presente estudio se halló una prevalencia de CAT de $73,6 \%$. Esta prevalencia resultó similar a la de investigaciones como la de López-Ramos (2013), ${ }^{13}$ que encontraron una prevalencia de76,47\% deCAT en una población peruana de la provincia de Huaura; lo mismo sucede en la de Zambrano (2015), ${ }^{3}$ con $79,5 \%$ en Venezuela, Saldarriaga $(2010)^{16}$ en Colombia, con $74,9 \%$. Sin embargo, estos resultados difieren con los obtenidos por Aguilar-Ayala en México (2014) ${ }^{10}$ con 35\%,
Montero (2011), ${ }^{11}$ también en México, con $59,5 \%$, y con Fleming (2016), ${ }^{20}$ en Estados Unidos, con $17,7 \%$. Estas diferencias en los resultados podrían deberse a que en los diferentes estudios varía el lugar de procedencia de los participantes, los cuales pueden diferir en sus hábitos alimenticios, la higiene oral y las costumbres, que son factores de riesgo para la presencia de CAT.

En el presente trabajo de investigación se refleja la necesidad de los tratamientos más requeridos por los niños examinados, siendo el de mayor necesidad de atención 
las obturaciones, particularmente "una obturación superficial" y "dos a más obturaciones superficiales", seguido del tratamiento "sellante/preventivo". Al comparar estos resultados con los obtenidos por otros estudios, se encuentran similitudes con el de GómezOsorno (2015) $)^{12}$ que encontró que la mayor necesidad de tratamiento correspondía a "obturaciones" y "sellantes/flúor barniz"; lo mismo sucede con el estudio realizado por Ramírez-Puerta (2013) ${ }^{21}$ que encontró que la mayor necesidad de atención era para "tratamientos preventivos" y "obturaciones". No obstante, en estos dos estudios se encontró mayor prevalencia en la necesidad de tratamientos preventivos que en la de obturaciones. Por otro lado, los resultados difieren de los obtenidos por Guerrero (2015), ${ }^{22}$ quien encontró una mayor necesidad de tratamiento en las "coronas de acero", seguido de las "resinas compuestas" y los "sellantes de fosas y fisuras".

En cuanto a lo que indican los resultados obtenidos en el presente estudio en el cuestionario ECOHIS, la mayor parte de los padres o apoderados reportaron que sus hijos nunca perdieron actividades preescolares en la escuela. Este hallazgo puede estar relacionado con falta de prevención y promoción, pues si el niño no experimenta sintomatología, no se realiza una evaluación odontológica. La CAT severa muestra un impacto negativo en el niño en los dominios "síntomas orales", "limitaciones funcionales" y "aspectos psicológicos", y uno menos severo en "autoimagen/interacción social". En la época preescolar (3 a 5 años) el niño empieza a interactuar con su familia y con otras personas de su entorno, las cuales van a influenciarlo en el desarrollo de su personalidad y en su manera de pensar y de hacer las cosas. En cuanto al desarrollo emocional y social, es en esta etapa que el niño se compara con otros, desarrolla su identidad y muestra sus emociones en formas más complejas; comienza a entender las normas y los valores sociales y a demostrar cierto control de emociones como la vergüenza, el orgullo y el sentimiento de culpabilidad. ${ }^{23}$ Durante el desarrollo del niño la salud oral es muy importante, ya que unos dientes y una sonrisa saludables van a influir en su autoestima y a contribuir a desarrollar relaciones interpersonales positivas.

Asimismo, cabe mencionar que la CAT severa muestra un impacto negativo en el dominio "angustia de los padres" y "función familiar", en los que se refleja cómo la situación bucal del niño afecta al entorno familiar, al tener los mayores que ausentarse en sus labores, con lo que eso repercute en la economía familiar. Además, el sentimiento de culpa en los padres es alto, pero al mismo tiempo muchos de ellos no hacen nada por solucionar el problema, y esto se reflejó en la evaluación oral de los niños que participaron en el presente estudio.

Resultados similares fueron reportados por López (2013) $)^{13}$ que investigó calidad de vida y problemas bucales en preescolares en una población peruana, concluyendo en que la CAT severa presentó un impacto negativo en la salud oral del niño. En el mismo sentido, se observó un mayor impacto en "limitaciones funcionales" y "angustia de los padres". Por otro lado, tenemos resultados similares reportados por Abanto en el año 2011, ${ }^{24}$ quien observó un impacto negativo en los dominios del ECOHIS, siendo "limitaciones funcionales" y "angustia de los padres" los dominios de mayor impacto tanto en los niños como en 
la familia. Sin embargo, la investigación de Martins-Junior realizada en $2012^{25}$ muestra un impacto negativo de la CAT severa en los niños examinados, con un promedio menor de "limitaciones funcionales" y de "angustia de los padres", resultados que difieren del presente estudio. Esto se puede deber a que la población de MartinsJunior solo comprende a niños de entre 3 y 4 años y excluye a los de 5, quienes podrían verse más afectados por el tiempo de la enfermedad presente en boca. Cabe mencionar que, pese a que los estudios se han realizado en diversos lugares y si bien los dominios más afectados podrían variar, todos coinciden en que la calidad de vida se ve afectada por la salud oral de los menores.

Por otra parte, en la literatura se ha demostrado la relación entre las características sociodemográficas y la caries dental. Torres-Ramos (2015) en Perú, ${ }^{26}$ Martins-Junior (2013) en Brasil ${ }^{2}$ y Abanto (2011) también en Brasil ${ }^{24}$ reportaron que un nivel socioeconómico bajo repercute negativamente en la calidad de vida de los preescolares y sus familias, y es por ello que consideró como covariable la edad, el sexo y el nivel socioeconómico. Sin embargo, en la presente investigación no se encontró relación entre las características sociodemográficas y la calidad de vida relacionada a la salud bucal.

En los últimos años han surgido diversos estudios relacionados con la calidad de vida en las diferentes áreas de la salud; sin embargo, la literatura realizada en base a población peruana es muy escasa, por locual el presente estudio es de gran importancia como base para futuras investigaciones. Los resultados encontrados, en general, aportan hallazgos que argumentan que la severidad de caries y la necesidad de tratamiento pueden provocar un impacto negativo en la calidad de vida del niño y la familia. En tal sentido, es vital la realización de nuevas investigaciones para fortalecer los conocimientos y las actitudes de los padres.

\section{Conclusión}

Del presente estudio se concluye que la alta prevalencia de CAT y el gran porcentaje de necesidad de tratamientos tienen una estrecha relación con la calidad de vida, lo que resulta en un impacto negativo para los menores. Por este motivo, se ve la necesidad de crear políticas de intervención que prevengan la enfermedad, detengan la progresión y traten las secuelas de la caries dental de modo que no exista una alta demanda de tratamientos dentales y que la calidad de vida de los menores no se vea afectada, para que puedan desenvolverse de manera óptima. Por otro lado, se recomienda continuar este estudio con niños de educación primaria y secundaria, de modo de evidenciar el impacto de la enfermedad en dichas poblaciones.

\section{Referencias bibliográficas}

1. World Health Organization Oral Health. "Salud bucodental", nota informativa 318. [Internet]. 2012 [consultado el 26 de julio de 2018]. Disponible en http://www.who.int/mediacentre/factsheets/fs318/es/.

2. Moreno X, Vera C, Cartes-Velásquez R. Impacto de la salud bucal en la calidad de vida de escolares de 11 a 14 años, Licantén, 2013. Rev Clín. Periodoncia Implantol Rehabil Oral. 2014; 7(3): 142-148.

3. Zambrano O, Fong L, Rivera L, Calatayud E, Hernández J, Maldonado A, Rojas-Sánchez F, Principe S, Pérez G, Navarro T, Acevedo A. Impacto de la caries de infancia temprana en la calidad de vida del niño zuliano y su familia. Odous Científica. 2015; 16(2): 8-17.

4. Urzúa A, Caqueo-Urizar A. Calidad de vida: una revisión teórica del concepto. Terapia Psicológica. 2012; 30(1): 61-71.

5. Muñoz P, Aguilar-Díaz F, De la Fuente J, Shimada H, Acosta-Torres L. Instrumentos validados para medir la salud bucal en niños. Salud Ciencia. 2014; 20: 846-851. 
6. Ministerio de Salud del Perú. Guía Práctica Clínica para la Prevención, Diagnóstico y Tratamiento de la Caries Dental en Niñas y Niños. Resolución Ministerial N 422-2017/Minsa.

7. Estrategias Sanitarias. Salud Bucal. [página en Internet]. Ministerio de Salud del Perú. Minsa; 2017. Disponible en: http://www.minsa.gob.pe.

8. American Academy of Pediatric Dentistry. Policy on Early childhood Caries (ECC): Classifications, Consequences and Prevention Strategies. Pediatr Dent. 2016; 38: 52-54.

9. Ghazal T, Levy SM, Childers NK, Broffitt B, Cutter G, Wiener HW, Kempf M, Warren J, Cavanaugh J. Prevalence and Incidence of Early Childhood Caries among African-American Children in Alabama. J Public Health Dent. 2015; 75(1): 42-48.

10. Aguilar-Ayala FJ, Duarte-Escobedo CG, Rejón-Peraza ME, Serrano-Piña R, Pinzón-Te AL. Prevalencia de caries de la infancia temprana y factores de riesgo asociados. Acta Pediat Mex . 2014; 35: 259-266.

11. Montero D, López P, Castrejón RC. Prevalence of early childhood caries and socioeconomical level. Revista Odontológica Mexicana. 2011; 15(2).

12. Gómez-Osorno AM, Bernal-Álvarez T, Posada-López A, Agudelo-Suárez AA. Caries dental, higiene bucal y necesidades de tratamiento en poblaciones de 3 a 5 años de una institución educativa de Medellín y sus factores relacionados. Rev Nac Odontol. 2015;11(21):23-35.

13. López-Ramos R, García-Rupaya C. Calidad de vida y problemas en preescolares de la provincia de Huaura, Lima. Revista Estomatológica Herediana. 2013; 23(3): 139-147.

14. Vera-Romero F, Vera-Romero O. Evaluación del nivel socioeconómico: presentación de una escala adaptada en una población de Lambayeque. Revista del Cuerpo Médico del HNAAA. 2013; 6(1): 41-45.

15. López-Ramos R, García-Rupaya C, Villena-Sarmiento R, Bordoni NE. Cross Cultural Adaptation An Validation of the Early Childhood Health Impact Scale (ECOHIS) in Peruvian Preschoolers. Acta Odontol. Latinoam. 2013; 26(3): 60-67.

16. Saldarriaga A, Arango CM, Cassio M. Dental Caries in the primary dentition of a Colombian population according to the ICDAS criteria. Braz Oral Res. 2010; 24(2): 211-6.

17. Pahel BT, Rozier GR, Slade GD. Parental perceptions of Children's oral health: The Early Childhood Oral Health Impact Scale. Health Qual Life Outcomes. 2007; 30(5): 6.

18. Sajadi SF, Pishbin L, Azhari SH, Moosazadeh M. Impact of oral and dental Health on Children's and Parents'quality of life Based on Early Childhood Oral Health Impact Scale (ECOHIS) Index. IJDSR. 2015;3(2):28-31.

19. World Health Organization. WHO Expert Consultation on Public Health Intervention against Early Childhood Caries Report of a Meeting. 2016.

20. Fleming E, Afful J. Prevalence of Total and Untreated Dental Caries Among Youth: United Sates, 20152016. U.S. Department of Health and Human Services. 2018; 307.

21. Ramírez-Puerta BS, Escobar-Paucar G, Castro-Aguirre J, Franco-Cortés AM. Necesidad de tratamiento en dentición primaria en niños de uno a cinco años con caries dental no tratada, en una comunidad de bajos ingresos. Moravia, Medellín, 2006. Rev Fac Odontol Univ Antioq 2009; 20(2): 129-137.

22. Guerrero MP, Aguilar EG, Gutiérrez JF, Lara IM. Prevalencia de caries temprana de la infancia y necesidad de tratamiento en niños que asisten a la clínica de la Especialidad en Odontopediatría de la Especialidad en Odontopediatría de la Universidad Autónoma de Nayarit (México). Rev Latinoam Orto Odontoped. 2015.

23. Gobierno de Navarra. El desarrollo psicológico del niño de 3 a 6 años. [página de internet]. Departamento de Educación. [fecha de acceso 10 de abril 2018]. España. Disponible en: https://www.educacion.navarra. es/documents/713364/714655/desarrollo.pdf/298a5bed-2c05-4bcb-b887-7df5221d6a1e.

24. Abanto J, Carvalho T, Mendes F, Wanderley M, Bönecker M, Raggio D. Impact of oral diseases and disorders on oral-health-related quality of life of preschool children. Community Dent Oral Epidemiol. 2011; 39(2): 105-114.

25. Martins-Junior PA, Vieira-Andrade RG, Corrêa-Faria P, Oliveira-Ferreira F, Marques LS, Ramos-Jorge ML. Impact of Early Childhood Caries on the Oral Health-Related Quality of Life of Preschool Children and Their Parents. Caries Res. 2013; 47:211-218.

26. Torres-Ramos G, Blanco-Victorio D, Chávez-Sevillano M, Apaza-Ramos S, Antezana-Vargas V. Impacto de la caries de la infancia temprana en la calidad de vida relacionada a la salud bucal en niños peruanos menores de 71 meses de edad. Odontología Sanmarquina. 2015; 18(2): 87.

Recibido: 11/10/2019

Aceptado: 05/01/2020

Correspondencia: Luis Angel Hayakawa Lastarria correo: luisangelhl@hotmail.com 